\title{
INFLUENCIA DE DOS TIPOS DE DIETAS SOBRE LA TALLA Y EL PESO CORPORAL EN NEONATOS DE CROCODYLUS ACUTUS CUVIER, 1807 (CROCODYLIDAE: CROCODYLIA) DEL ZOOCRIADERO DE MANZANILLO, CUBA
}

\author{
Mario PÉRez Gómez ${ }^{1}$, Carlos NARANJo LóPEZ², Bernardo ReYes TuR ${ }^{2}$ \\ e Irene VEGA RAMÍREZ 2 \\ ${ }^{1}$ Empresa Nacional para la conservación de La Flora y La Fauna de Cuba. Manzanillo, Provincia \\ Granma. CUBA \\ 2Universidad de Oriente. Calle $8 \mathrm{~N}^{\circ} 205$ e/n 7 y 9. Reparto Vista Alegre. Santiago de Cuba. CUBA \\ amaury@cedaimzo.co.cu naranjo@cnt.uo.edu.cu
}

Pérez Gómez, M., C. Naranjo López, B. Reyes Tur e I. Vega Ramírez. 2009. Influencia de dos tipos de dietas sobre la talla y el peso corporal en neonatos de Crocodylus acutus Cuvier, 1807 (Crocodylidae: Crocodylia) del Zoocriadero de Manzanillo, Cuba. Acta Zool. Mex. (n. s.) 25(1): 151-160.

RESUMEN. Se diseñó un experimento con 90 neonatos del cocodrilo americano, Crocodylus acutus, que fueron criados y alimentados con una dieta patrón por 50 días y dos dietas experimentales por 30 días, en tres grupos (A, B, C) mantenidos por separado en corrales en el Zoocriadero de Manzanillo (Provincia Granma) así como el costo de las mismas. Se analizó la influencia de dos tipos de dietas contra la dieta patrón sobre la talla y el incremento del peso corporal en $C$. acutus, así como el costo de las mismas. La dieta con pescados marinos (B) es más efectiva que la dieta patrón compuesta de pescados, hígado y pulmón de res (A) y que la de larvas de moscas (C), ya que la tasa de crecimiento de los neonatos fue de $3.5 \mathrm{~mm} /$ día. Los ejemplares con la dieta $\mathrm{B}$ crecieron 1.35 veces más con un incremento en peso de $3.71 \mathrm{~g} /$ día, valores superiores a los correspondientes a las otras dos dietas que incluyó la patrón. Se demostró que con la dieta $\mathrm{B}$ los neonatos crecen más que con las dietas A y $\mathrm{C}$, sin embargo cada neonato alimentado con la dieta B cuesta 14 veces más que con las otras dos.

Palabras Claves: Crocodylus acutus, cocodrilo americano, dieta de neonatos.

Pérez Gómez, M., C. Naranjo López, B. Reyes Tur \& I. Vega Ramírez. 2009. Influence of two types of diets on size and body weight in Crocodylus acutus, Cuvier 1807 neonates (Crocodylidae: Crocodylia) of Manzanillo Nursery, Cuba. Acta Zool. Mex. (n. s.) 25(1): 151-160.

ABSTRACT. An experiment was designed with 90 neonates of the American crocodile, Crocodylus acutus, that were raised and fed with a standard diet during 50 days and with two experimental diets for 30 days, in three groups (A, B, C) kept in experimental square tanks during 30 days at the Manzanillo Zoocriadero (Province of Granma). The influence on size and body weight increase of two types of diets against the standard diet in C. acutus was analyzed, as well as the economic costs associated. The sea fish diet (B) is more effective compared to the standard diet of fish, cow liver and lung (A) and to the fly maggot diet (C), since the growth rate of neonates was $35 \mathrm{~mm} /$ day, growing 1.35 times more. The weight increase was $3.71 \mathrm{~g} /$ day, higher than the increases obtained 
with the other two diets. It was demonstrated that with diet B neonates grew 1.35 times more than with the diets A and C. However, each neonate fed with diet B costs 14 times more than those fed with the other two diets.

Key Words: Crocodylus acutus, American crocodile, neonate diet.

\section{INTRODUCCIÓN}

El cocodrilo americano, Crocodylus acutus, se distribuye desde México hasta Perú por la costa Atlántica, y desde el sur de la Florida hasta Venezuela (Escobedo \& Mejía 2003a, 2003b, 2003c). Alcanza una longitud total promedio entre 300 y 400 $\mathrm{cm}$, aunque se han visto individuos de $700 \mathrm{~cm}$ (Thorbjarnarson 1989). Es una de las especies de crocodílidos de gran importancia comercial por su tamaño, excelente carne y magnífica piel con la cual se confeccionan múltiples piezas de vestuario, poseyendo además interés ornamental. Se considera que esta especie tiene un alto potencial para la cría en cautiverio (Chirivi 1973) con fines comerciales en Tanzania, Papúa-Nueva Guinea, Estados Unidos, Colombia y México figuran entre los países en los que existen programas encaminados a la protección y cultivo comercial de cocodrilos (Chabreck 1967, Pooley 1971, Joanen \& Mc Nease 1979, Umpiérrez et al. 1993, Àlvarez del Toro \& Sigler 2001).

El estatus de conservación de C. acutus es de vulnerable (Ross 1998). Las principales causas que amenazan a $C$. acutus en Cuba son la caza indiscriminada y sostenida, en décadas pasadas, así como la destrucción y contaminación de sus lugares de reproducción y alimentación.

En Cuba se realizan ingentes esfuerzos por salvar a esta especie de cocodrilo, por los que se han construido y operan en la actualidad siete criaderos de C. acutus distribuidos desde las provincias de Pinar del Río hasta Granma, en los que se desarrollan importantes acciones con el fin de mantener y/o aumentar sus poblaciones. Las actividades fundamentales de estos criaderos están dirigidas a favorecer las condiciones de vida, desde el cuidado postnatal hasta el manejo de los individuos reproductores. No obstante uno de los aspectos que presenta mayores dificultades en el manejo de la especie es la alimentación, debido a que el alimento consumido debe ser obtenido de productos que forman parte de la alimentación humana.

Entre los factores determinantes para el éxito en la cría de crocodílidos, figuran la densidad de cultivo, la dieta y la frecuencia de alimentación utilizada (Casas 1962). Existe poca información precisa disponible en relación con el crecimiento en cautiverio basada en la alimentación de estos reptiles. En Venezuela se han realizado ensayos de crecimiento para determinar la influencia de tres dietas a igual densidad de individuos de Caiman crocodilus, para los primeros cinco meses de vida (Pérez 2000). Rodríguez (1989) trabajó con neonatos y juveniles de C. fuscus de Colombia en tres modelos de crecimiento con variación de la dieta y la frecuencia de alimentación, mientras que Rodríguez \& Rodríguez (1989) evaluaron el crecimiento de neonatos y juveniles de C. acutus en cautiverio. 
En el Zoocriadero de Manzanillo (Provincia Granma) la alimentación de los neonatos de $C$. acutus se lleva a cabo mediante una dieta patrón, consistente en pulmón e hígado de res y pescado marino, durante el primer año de vida y especialmente en los primeros tres meses. Esta dieta no es constante lo que depende de la disponibilidad de cada uno de los productos utilizados.

Se llevó a cabo un experimento con el objetivo de evaluar el efecto de dos tipos de dietas en comparación con la dieta patrón sobre el peso y el tamaño de neonatos de $C$. acutus, de 20 días de nacidos hasta los dos meses de edad, así como para analizar los costos de las tres dietas.

\section{MATERIAL Y MÉTODOS}

La investigación se llevó a cabo en el zoocriadero de Manzanillo ubicado a $10 \mathrm{~km}$ al sureste de la ciudad de Manzanillo, en la región oriental de Cuba a orillas del Golfo de Guacanayabo.

Se mantuvieron tres grupos de neonatos de C. acutus en corrales, construidos con muros de cemento y tapados con una malla de color negro con función protectora que contribuye a elevar la temperatura. Cada corral presenta un largo de $3.25 \mathrm{~m}$ y un ancho de $2.25 \mathrm{~m}$ (área de $7.31 \mathrm{~m}$ ?). En cada corral la proporción tierra/agua fue de $50 \%$.

Los huevos utilizados en el experimento se obtuvieron de los corrales de reproducción en el zoocriadero. El período de incubación comenzó el 25 de febrero de 2007, con una duración de 90 a 100 días. Los nacimientos comenzaron el día 25 y 26 de mayo de 2007 y a partir de 10 días después de la eclosión se eligieron los neonatos.

El experimento consistió de los siguientes pasos:

1. Preparación de corrales de cría (A, B y C) para albergar tres grupos de 30 neonatos en cada uno, para un total de 90 individuos. Cada corral fue escogido con similares condiciones de construcción, temperatura, iluminación y cobertura, tratando de amortiguar cualquier factor alineado que pudiera influir sobre el experimento.

2. Preparación de los tres tipos de dietas: La dieta A se consideró como patrón y fue confeccionada a base de pescados marinos, hígado, y pulmón de res, mezclados en proporción de 1:1:1. Esta dieta fue ofrecida al grupo A durante todo el experimento con individuos de 20 días de nacidos. La dieta $\mathrm{B}$ se confeccionó con pescado marino exclusivamente, se proporcionó al grupo $\mathrm{B}$, compuesto por individuos de 20 días de edad. Se conoce que esta dieta es la más cara, pero se experimentó con ella debido a que en numerosas ocasiones no se cuenta con la dieta patrón. La dieta $\mathrm{C}$ se compuso de larvas de moscas enriquecida con calcio y fue ofrecida al grupo C integrado por individuos de 20 días de edad. Esta dieta se confeccionó por iniciativa de uno de los autores. 
3. Registro de medidas y peso. La longitud total de los individuos se midió con una cinta métrica con precisión de un milímetro y el peso se obtuvo con balanzas Pesola de 100 y 1000 g. A partir de la eclosión (26/4/2007) transcurrieron 10 días $(6 / 5 / 07)$ para la primera medición del largo y peso de 90 neonatos escogidos mediante un muestreo al azar. Estos individuos no recibieron alimento durante los primeros 10 días, debido a que disponen de suficientes reservas energéticas después de la eclosión. La segunda medición de la longitud y el peso se efectuó a los 20 días de eclosionados los huevos (16/5/07) y se excluyeron los individuos medidos en la primera ocasión. En esta ocasión se escogieron 90 neonatos definitivos para el experimento mediante un muestreo selectivo por tallas, obteniéndose para cada uno de los tres grupos experimentales 10 individuos de $31 \mathrm{~cm}, 10$ de $32 \mathrm{~cm}$ y 10 de 33 $\mathrm{cm}$ de longitud total. Los individuos se midieron y pesaron después que fueron alimentados uniformemente durante diez días en los tres corrales con la dieta patrón, con el objeto de estabilizar la mortalidad inicial en caso de que se suministraran dietas no apropiadas en la experimentación.

A partir de la tercera medición de la longitud y del peso hasta la quinta (desde 20 hasta 50 días después de eclosionados, 26/6/07, 6/7/07 y 16/7/07), se mantuvo cada grupo con su dieta respectiva y hubo una diferencia de 10 días entre cada una de las cinco mediciones y pesajes.

El suministro del alimento se efectuó siempre a las 2:00 pm con el objetivo de aprovechar la temperatura máxima a esta hora del día, aspecto muy importante para la obtención de calor necesario en la alimentación, debido a que los cocodrilos tienen una digestión lenta. El suministro del alimento fue diario y por la misma persona, se proporcionaron $27 \mathrm{~g}$ de alimento por neonato.

Análisis de los datos. Para el análisis estadístico de los datos obtenidos se determinó que el factor de diseño del experimento fuera la dieta. Como estadísticos de la longitud total y el peso de los individuos mantenidos bajo cada una de las tres dietas se utilizaron la media, la desviación estándar (DE) y el coeficiente de variación (CV en \%).

A partir de los datos de la tercera medición de los individuos se llevó a cabo el análisis de la variación de la talla y el peso, debido a que en la segunda medición los neonatos fueron inicialmente alimentados con la misma dieta patrón a partir de que alcanzaron una longitud promedio de $32 \mathrm{~cm}$. Se probaron las diferencias entre las tallas y pesos obtenidas en mediciones sucesivas en relación con las dietas aplicadas utilizando análisis de varianza (ANOVA) completamente aleatorizado y se aplicó la prueba de Duncan para la comparación de medias a posteriori $(\mathrm{P} \leq 0.05)$, por medio del programa MINITAB Statistical Software, Release 13.30.

Se estimaron las tasas de crecimiento corporal ( $\mathrm{mm} /$ día) y de incremento del peso (g/día) de los individuos de acuerdo con las tres dietas empleadas calculando el promedio de talla o peso entre mediciones sucesivas, desde la segunda hasta la quinta 
medición, obteniéndose tres valores que fueron divididos entre 30 días, periodo en el que se les suministraron las tres dietas.

Se calculó el costo de alimentación de cada neonato durante los 50 días que duró el experimento, de los cuales no se alimentaron los primeros 10 días, los 10 días siguientes se alimentaron los tres grupos con la dieta patrón y los restantes 30 días con los dos tipos de dietas (B y C) y la dieta patrón (A).

\section{RESULTADOS}

Variación de la talla. La mayor diferencia en talla media de los neonatos desde la inicial a la final se registró en el grupo mantenido con la dieta $\mathrm{B}$ con incremento total de $17.2 \mathrm{~cm}$. En orden decreciente le siguieron el grupo A y C con $14.4 \mathrm{~cm}$ y 13.2 $\mathrm{cm}$, respectivamente. En los tres tipos de dietas, los coeficientes de variación son menores en el último muestreo si se comparan con la variación inicial del primer muestreo (Cuadro 1).

Al comparar el efecto del tipo de dieta sobre la talla se detectaron diferencias estadísticamente significativas dentro de la cuarta $(\mathrm{F}=115.91, \mathrm{p}<0.001)$ y quinta medición ( $\mathrm{F}=189.06, \mathrm{p}<0.001$; Cuadro 1). La prueba de Duncan indicó que existen diferencias entre todas las medias comparadas. En el caso de la tercera medición aunque también existen diferencias significativas $(\mathrm{F}=7.45 ; \mathrm{p}=0.001)$, la prueba de Duncan sólo muestra diferencias entre la media de la talla de los individuos que consumieron pescado (Dieta B) con aquellos que se alimentaron de larvas de mosca (Dieta C) y la dieta patrón (Dieta A).

Cuadro 1. Mediciones sucesivas de longitud total (media en $\mathrm{cm} \pm 1$ D.E., CV\% en paréntesis, $\mathrm{n}=30$ en todos los casos) de neonatos de Crocodylus acutus sometidos a 3 dietas diferentes (ver texto). Los supraíndices muestran la igualdad (iguales) o diferencias (letras diferentes) entre las medias de grupos de tratamientos dentro de la misma medición de acuerdo con la prueba de Duncan.

\begin{tabular}{lccccc}
\hline \multicolumn{5}{c}{ Medición } \\
\hline Dieta & 1 & 2 & 3 & 4 & 5 \\
A & $25.8 \pm 1.8(7.0)^{\mathrm{a}}$ & $32 \pm 0.9(2.8)^{\mathrm{a}}$ & $32.8 \pm 0.9(2.7)^{\mathrm{a}}$ & $36.0 \pm 0.9(2.5)^{\mathrm{b}}$ & $40.2 \pm 0.8(2.0)^{\mathrm{b}}$ \\
B & $25.7 \pm 1.8(7.0)^{\mathrm{a}}$ & $32 \pm 0.8(3.1)^{\mathrm{a}}$ & $33.5 \pm 1.2(3.6)^{\mathrm{b}}$ & $38.3 \pm 0.7(1.8)^{\mathrm{c}}$ & $42.9 \pm 0.8(1.9)^{\mathrm{c}}$ \\
C & $25.7 \pm 1.7(6.6)^{\mathrm{a}}$ & $32 \pm 0.8(2.5)^{\mathrm{a}}$ & $32.6 \pm 0.6(1.8)^{\mathrm{a}}$ & $35.3 \pm 0.7(2.0)^{\mathrm{a}}$ & $38.9 \pm 0.8(2.1)^{\mathrm{a}}$ \\
\hline
\end{tabular}

Variación del peso corporal. La mayor diferencia en peso corporal promedio de los neonatos desde la inicial a la final se detectó en el grupo mantenido con la dieta B con incremento total de $167.4 \mathrm{~g}$. En orden decreciente le siguieron el grupo A y C con 142.9 g y 135.3 g, respectivamente. Los coeficientes de variación de las mediciones 4 y 5 son menores que las primeras tres mediciones (Cuadro 2). 
Cuadro 2. Mediciones sucesivas de peso corporal (media en $\mathrm{cm} \pm 1$ D.E., CV\% en paréntesis, $\mathrm{n}=30$ en todos los casos) de neonatos de Crocodylus acutus sometidos a 3 dietas diferentes (ver texto). Los supraíndices muestran la igualdad (letras iguales) o diferencias (letras diferentes) de acuerdo con la prueba de Duncan entre las medias de grupos de tratamientos dentro de la misma medición.

\begin{tabular}{cccccc}
\hline & \multicolumn{5}{c}{ Medición } \\
\hline Dieta & 1 & 2 & 3 & 4 & 5 \\
& $59.4 \pm 5.5$ & $72.1 \pm 17.1$ & $118.0 \pm 17.1$ & $156.2 \pm 9.2$ & $202.3 \pm 8.6$ \\
A & $(9.3)^{\mathrm{a}}$ & $(23.7)^{\mathrm{a}}$ & $(14.5)^{\mathrm{a}}$ & $(5.9)^{\mathrm{a}}$ & $(4.3)^{\mathrm{b}}$ \\
& $58.3 \pm 6.6$ & $80.0 \pm 12.4$ & $120.3 \pm 15.5$ & $177.5 \pm 9.1$ & $225.7 \pm 7.9$ \\
B & $(11.3)^{\mathrm{a}}$ & $(15.5)^{\mathrm{a}}$ & $(12.9)^{\mathrm{a}}$ & $(5.1)^{\mathrm{b}}$ & $(3.5)^{\mathrm{c}}$ \\
& $54.9 \pm 7.3$ & $81.3 \pm 11.2$ & $115.2 \pm 12.4$ & $157.7 \pm 7.0$ & $190.2 \pm 4.6$ \\
C & $(13.3)^{\mathrm{a}}$ & $(13.8)^{\mathrm{a}}$ & $(10.8)^{\mathrm{a}}$ & $(4.4)^{\mathrm{a}}$ & $(2.4)^{\mathrm{a}}$ \\
\hline
\end{tabular}

Al comparar el efecto del tipo de dieta sobre el peso corporal en la tercera medición no hubo diferencia significativa $(\mathrm{F}=0.88, \mathrm{p}=0.419)$, pero si se detectaron diferencias significativas en la cuarta $(\mathrm{F}=59.13, \mathrm{p}<0.001)$ y quinta medición $(\mathrm{F}=186.79, \mathrm{p}<0.001)$. En el caso de la cuarta medición, la prueba de Duncan sólo mostró diferencias entre la media del peso de los individuos que consumieron pescado (Dieta B) con aquellos que se alimentaron con larvas de mosca (Dieta C) y con la dieta patrón (Dieta A). La prueba de Duncan indicó que existen diferencias entre todas las medias comparadas para la quinta medición.

Tasas de incremento de talla y peso. El grupo $\mathrm{B}$, alimentado con pescado, mostró los mayores valores de la tasa de crecimiento, $3.67 \mathrm{~mm} /$ día, y de incremento del peso, $5.47 \mathrm{~g} /$ día, seguido del grupo A, alimentado con la dieta patrón, con 2.73 $\mathrm{mm} /$ día y $4.43 \mathrm{~g}$ /día, y del grupo $\mathrm{C}$, alimentado con larvas de moscas, con 2.30 $\mathrm{mm} /$ día y $3.63 \mathrm{~g} /$ día, respectivamente (Fig. 1).

Costo de las dietas. Se alimentaron un total de 90 neonatos a razón de $27 \mathrm{~g}$ de alimento diario cada uno, en los tres corrales se gastaron $2.43 \mathrm{~kg}$ diarios, $17.01 \mathrm{~kg}$ semanales y 72.9 mensuales (Cuadro 4). De los 50 días que duró el estudio no se proporcionó alimento los 10 primeros días. Durante todo el experimento se consumieron $97.2 \mathrm{~kg}$.

El costo final de la alimentación de cada neonato para las tres dietas fue de $\$ 0.30$ (dieta A, patrón), con la dieta de pescado fue de $\$ 2.44$ (dieta B) y con la dieta de larvas de mosca (dieta C) fue de $\$ 0.18$ (Cuadro 3). 


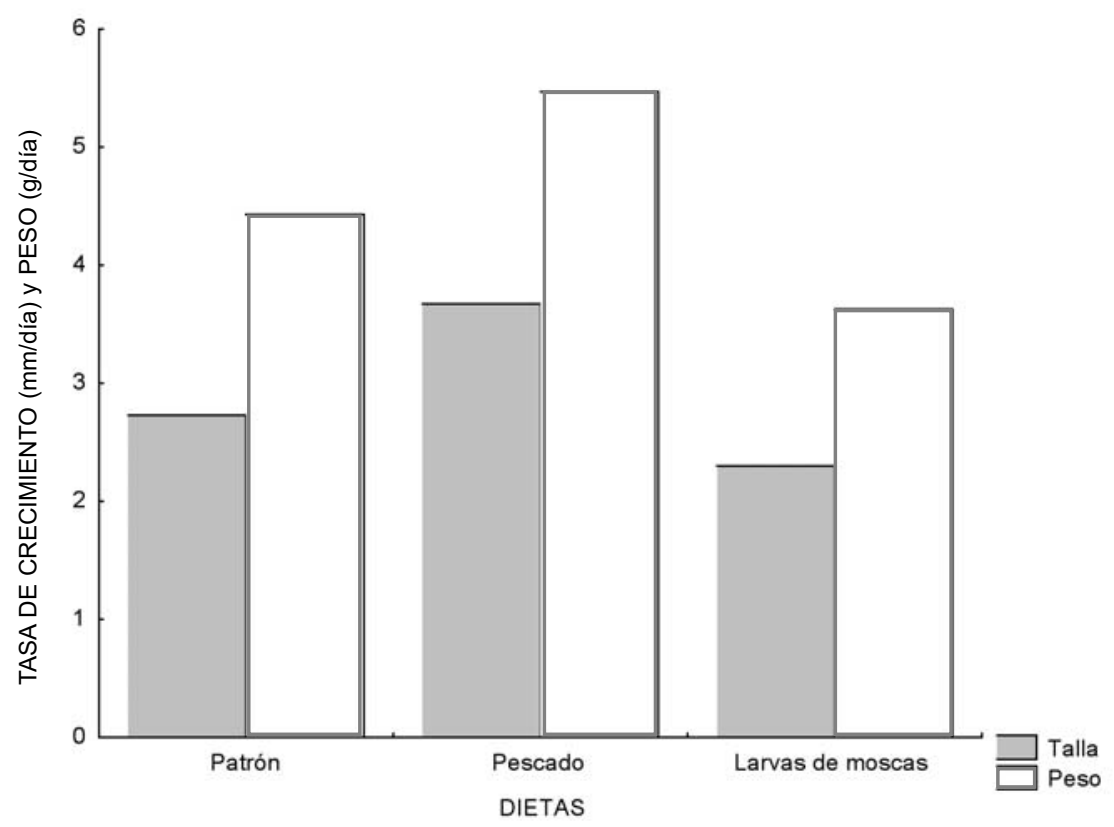

Figura 1. Tasas de incremento diario de la talla y peso corporal en neonatos de Crocodylus acutus alimentados con tres dietas.

\section{DISCUSIÓN}

Variación de la talla. Las diferencias estadísticamente significativas entre los tres grupos de tratamiento, sugieren que el tamaño corporal de los neonatos presentó una alta sensibilidad al cambio de dietas durante el experimento, lo cual valida el efecto diferencial de la calidad del alimento sobre el crecimiento de los individuos.

Sólo en el caso de los valores de las terceras mediciones de los grupos A (con dieta patrón) y C (con larvas de moscas) no hubo diferencias significativas (Cuadro 1). Esto podría deberse a que en la segunda medición los neonatos escogidos tuvieron el mismo promedio de largo $(32 \mathrm{~cm})$ y sólo fueron alimentados durante 10 días con los tres tipos de dietas y posiblemente en este corto tiempo no se expresa un efecto diferencial sobre el tamaño de los neonatos. Sin embargo, en el caso del grupo B (alimentados con pescado) ya desde esta temprana medición hay diferencias significativas, lo cual indica alta efectividad de la dieta de pescado influyendo sobre el tamaño de los neonatos, pero se hace necesario tener en cuenta que una alimentación prolongada (después de tres meses) con pescado puede resultar no adecuada (Lance, 1987). 
Variación del peso corporal. De las comparaciones que no presentaron diferencias significativas, tres están incluidas en la tercera medición (Cuadro 2), lo que se puede interpretar como la ausencia de un efecto del cambio de dieta en los primeros 10 días de ser alimentados de forma diferenciada. Adicionalmente, en la cuarta medición también se detectaron medias estadísticamente iguales, este patrón demuestra que el efecto de las dietas sobre el peso se expresa claramente a partir de la quinta medición, a diferencia de la talla donde se detectó desde la cuarta medición.

Tasas de incremento de talla y peso. El grupo B, alimentado solamente con pescado, presentó una tasa de crecimiento mayor en comparación con el grupo A alimentado con la dieta patrón, y el grupo $\mathrm{C}$, alimentado con larvas de moscas. Los neonatos del grupo B crecieron diariamente 1.3 veces más que los del grupo A y 1.6 más que los del grupo C. Para el peso ocurrió exactamente lo mismo, los neonatos del grupo B pesaron 1.3 veces más que los del grupo A y 1.6 más que los del grupo C. El incremento de la talla y el peso de los neonatos poseen una relación directa durante los primeros 50 días de vida, dadas las semejanzas en las tendencias de ambas variables entre los tres grupos (Fig. 1).

Los resultados de la tasa de crecimiento sugieren que la dieta con pescado es más efectiva que la dieta patrón y la de larvas de moscas. Casas \& Barrios (2003) determinaron que en las heces fecales de C. acutus silvestres en México un porcentaje alto estaba representado por peces $(30.35 \%)$ y crustáceos $(26.78 \%)$. Por tanto, si se les suministra artificialmente una dieta a base de pescado como fue en el grupo B, podría haber un efecto positivo sobre el desarrollo de los neonatos. Diferentes autores han calculado las tasas de crecimiento en juveniles de C. acutus. Thorbjarnarson (1989) encontró que la tasa de crecimiento en el orden Crocodylia es durante los tres primeros meses de vida y disminuye a partir del primero o segundo año, en dependencia de la especie; Velasco \& Lander (1998) reportaron $8.4 \mathrm{~mm} /$ día en condiciones silvestres; Chabreck \& Joanen (1979) de 1 a $4 \mathrm{~mm} /$ día en condiciones silvestres; Pérez (2000) 1-3.9 mm/día en cautiverio, Rodríguez \& Rodríguez (1989) $1 \mathrm{~mm} /$ día en cautiverio y Dowling \& Brazaitis (1966) $0.9 \mathrm{~mm} /$ día en cautiverio. En el presente trabajo se detectó una tasa de crecimiento mínima de $2.30 \mathrm{~mm} /$ día y una máxima de $3.67 \mathrm{~mm} /$ día (Fig. 1), resultados intermedios entre los valores encontrados por los autores mencionados, lo que ratifica que las condiciones de cría y la alimentación en nuestro experimento fueron propicias para los neonatos.

Con respecto a las tasas de incremento del peso corporal, los resultados son muy similares a los alcanzados con la talla y corroboran la efectividad de la dieta de pescado con respecto a la dieta patrón y a la dieta con larvas de moscas.

Costo de las dietas. La dieta $\mathrm{B}$ a base de pescado es la más efectiva ya que los neonatos crecen 1.35 veces más que en las otras dos dietas (la patrón y la de larvas de moscas), pero cada neonato criado con esta dieta cuesta casi 14 veces más que si fueran criados con larvas de moscas y 8 veces más con la dieta patrón (Cuadro 3). Si 
tenemos en cuenta estas cifras se puede recomendar la dieta de larvas de moscas como un medio de abaratar la crianza de los neonatos en condiciones de cautiverio. Sin embargo, no se conoce el efecto que a largo plazo pudiera causar este tipo de dieta sobre el tamaño, el peso, la conducta y la fisiología de los organismos.

Cuadro 3. Cantidad de alimento ingerido por neonatos de Crocodylus acutus en 40 días sometidos a 3 dietas diferentes (ver texto) y su costo en moneda nacional.

\begin{tabular}{cccccccc}
\hline Dieta & $\begin{array}{c}\mathrm{N}^{\mathrm{o}} \mathrm{de} \\
\text { Individuos }\end{array}$ & Tiempo & $\begin{array}{c}\text { Precio/ } \\
\mathrm{kg}\end{array}$ & $\begin{array}{c}\text { Peso en } \\
\mathrm{kg}\end{array}$ & Costo & $\begin{array}{c}\text { Costo/ } \\
\text { Grupo }\end{array}$ & $\begin{array}{c}\text { Costo/ } \\
\text { Neonato }\end{array}$ \\
\hline $\mathrm{A}$ & 30 & 40 días & 0.28 & 32.4 & $\$ 9.07$ & $\$ 9.07$ & $\$ 0.30$ \\
$\mathrm{~B}$ & 30 & 10 días & 0.28 & 8.1 & $\$ 2.27$ & & \\
& & 30 días & 2.92 & 24.3 & $\$ 70.96$ & $\$ 73.23$ & $\$ 2.44$ \\
$\mathrm{C}$ & 30 & 10 días & 0.28 & 8.1 & $\$ 2.27$ & & \\
& & 30 días & 0.14 & 24.3 & $\$ 3.40$ & $\$ 5.67$ & $\$ 0.18$ \\
Total & 90 & & & 97.2 & $\$ 87.97$ & $\$ 87.97$ & \\
\hline
\end{tabular}

Una solución que podría aplicarse teniendo en cuenta estos resultados es hacer un plan de manejo alimentario que incluya la dieta con larvas de mosca de forma rotativa, de manera tal que los neonatos no se alimenten permanentemente de dicha dieta, esto aliviaría desde el punto de vista económico la crianza, sin que se corra el riesgo de propiciar algún efecto no deseado en la fisiología reproductora de los individuos.

AGRADECIMIENTOS. A todo el personal del Zoocriadero de Manzanillo que de forma esmerada y dedicada ayudó con la alimentación y la toma de datos en los neonatos. A Amador Ruiz Baliú por la exigente revisión de la traducción del resumen en inglés. Las observaciones de los revisores anónimos permitieron mejorar sustancialmente el manuscrito.

\section{LITERATURA CITADA}

Álvarez del Toro, M. \& L. Sigler. 2001. Los Crocodylia de México. IMERNAR, PROFEPA. México. Casas, G \& G. Barrios. 2003. Hábitos alimenticios de Crocodylus acutus (Reptilia: Crocodylidae) determinados por el análisis de sus excretas en la costa de Jalisco, México. Anales del Instituto de Biología, Universidad Nacional Autónoma de México, Serie Zoología 74 (1): 35-42.

Chabreck, R. 1967. Alligator farming hunts. Wildlife and fisheries Commission, Miami, Florida.

Chabreck, R. \& T. Joanes. 1979. Growth rates of American Alligators in Louisiana. Herpetologica 35 : 51-57.

Chirivi, H. 1973. Contribución al conocimiento de la babilla o Yacaré Tinga (Caiman crocodilus) con notas acerca de su manejo y de otras especies de Crocodilia neotropicales. Proc. Simposio Int. sobre Fauna Silvestre y Pesca Fluvial y Lacustre Amazónica. Manaus, Brasil. 
Dowling, H. \& P. Brazaitis. 1966. Size and growth in captive crocodilians. International Zoo Yearbook 6: 256-270.

Escobedo, A. H. \& F. Mejía. 2003a. El «Cocodrilo de Tumbes» (Crocodylus acutus Cuvier, 1807): estudio preliminar de su estado actual en el norte de Perú. Ecología Aplicada 2(1): 133-135.

Escobedo, A. H. \& F. Mejía. 2003b. Distribución actual e histórica de Crocodylus acutus Cuvier, 1807 en Perú: Implicaciones para su Conservación. Memorias de la V Reunión del Subcomité Técnico para el Manejo y Aprovechamiento de los Crocodylia en México. ZOOMAT, Chiapas, México.

Escobedo, A, H. 2003. Períodos de actividad y efecto de las variables ambientales en cocodrilos (Crocodylus acutus Cuvier, 1807): evaluando los métodos de determinación de la fracción visible. Ecología Aplicada 2(1): 136-140.

Joanen, T \& L. Mc Nease. 1979. Culture of American Alligator. International Zoo Yearbook 19: 61-66.

Lance, V. 1986. Hormonal control of reproduction in crocodilians. Pp. 410-411 In: Grahame. Weeb et al. (eds). Wildlife management: crocodiles and alligators. Surreay Beatty and Sons Pty Ltd. in association with the Conservation Commission of the Northern Territory. Australia.

Pérez, A. 2000. Crecimiento del Caiman crocodilus crocodilus en cautiverio. Interciencia 25(9): 442446.

Pooley, A. 1971. Crocodile rearing and restocking. IUCN Publications New Series. Supplementary Paper 32: 104-130.

Rodríguez, A. E. \& M. Rodríguez. 1989. Evaluación del crecimiento y levante de neonatos y juveniles de Crocodylus acutus Cuvier, (Crocodylia: Crocodylidae) durante 1987-1988 en el centro experimental de fauna silvestre de San Marcos (Sucre). Trianea 3: 53-60.

Rodríguez, M. 1989. Tres modelos de crecimiento en longitud de neonatos y juveniles de Caiman crocodilus fuscus (Cope: 1968) (Crocodylia:Alligatoridae) en cautiverio. Trianea 3: 61-66.

Ross, J. P. 1998. Crocodiles. Status survey and conservation action plan. 2nd Edition. IUCN/SSC Crocodile Specialist Group. IUCN, Gland, Switzerland and Cambridge, UK.

Thorbjarnarson, J. B. 1989. Ecology of the American crocodile, Crocodylus acutus. Crocodiles. Their ecology, management, and conservation. IUCN Publications New Series: 229-259.

Umpiérrez, S., M. Santos, C. Ríos, N. Cundines \& F. Achaval. 1993. Estudio de alimentación en cautiverio de Caiman latirostris. Conselho Nacional de Desenvolvimiento Científico e Tecnológico. Sao Pablo, Brasil.

Velasco, A. \& A. Lander. 1998. Evaluation of the reintroduction program for American Crocodiles (Crocodylus acutus) in the Cuare wildlife refuge, Falcon State, Venezuela. Proceeding of the 14 th Working Meeting of the Crocodile Specialist Group-IUCN: 320-324.

Recibido:30 de noviembre de 2007

Aceptado: 7 de octubre de 2008 